\title{
SIMULATING LAYUP DEFECTS DURING TOW STEERING IN AUTOMATED FIBER PLACEMENT
}

\author{
Nima Bakhshi and Mehdi Hojjati \\ Concordia Center for Composites (CONCOM), \\ Concordia University, \\ Montreal, Canada.
}

\begin{abstract}
Automated Fiber Placement (AFP) offers valuable advantages which make this technology a suitable candidate for producing high-quality parts in the aerospace industry. However, there are limitations: number of defects may arise during the fiber placement including wrinkles at the inside edge, and blisters in the middle of the prepreg tows. These defects have severe effects on the layup quality and consequently, on the performance and quality of the final part. Therefore, efforts shall be pointed to avoid them. A deeper understanding of the defect formation processes as well as tools and techniques for modeling them is indispensable for fully harnessing the potential of AFP technology. In the present study, a physics-based modeling approach is presented for the global modeling of defects in AFP. The application of this approach for detecting and modeling the blisters and outof-plane wrinkles that appear during fiber steering, is discussed, although it should be noted that the application is not limited to the case of fiber steering and can be expanded to different scenarios. Preliminary results of the simulations are presented. AFP trials are performed to validate the model. The trends and patterns of both wrinkles and blisters are found to be in good agreement with experimental results.
\end{abstract}

Keywords - Automated Fiber Placement; layup defect; simulation; manufacturing.

\section{INTRODUCTION}

Automated Fiber Placement has been increasingly used in the aerospace industry to manufacture high-quality aerospace parts. Manufacturing objectives including increasing productivity and reproducibility as well as decreasing labor intensity and minimizing the number of parts can be accomplished using this technology. One of the unique features of AFP is its ability to deliver up to 32 prepreg tows in a course with different speeds. This feature namely, the differential tow pay-out is what enables the AFP machines to laminate complex geometries and Variable Stiffness Composites (VSC). In complex geometries, steering the fiber path away from the geodesic path of the mold to maintain the desired fiber orientation is inescapable. Moreover, differential tow pay-out allows engineers to design a family of composites with curvilinear fiber paths, called VSCs. A more efficient load path between supports and load points is formed by curvilinear fibers which allows exploiting the full potential of the directionality of composite laminae [1].

Despite its numerous advantages and capabilities, AFP comes with its own limitations. The productivity of the lamination process and the quality of final parts are significantly influenced by the complexity of the part, as well as the process parameters such as lay-up temperature and speed, compaction force and fiber tension. Several different defects may appear in AFP (especially during fiber steering) such as out-of-plane wrinkles at the inside edge of the tow, blisters (circular delaminations in form of a blister) in the middle of the tow, tow pull ups and shearing effects. These defects will cause lower part quality with reduced reproducibility by deviating the fibers from the original prescribed path, increasing resin rich areas, etc. Consequently, efforts must be aimed at avoiding them $[1,2]$.

The key mechanism resisting against the formation of most layup defects is prepreg tack [3-5]. Tack is a complex systemic property of prepreg-tool interface that can be reasonably controlled by process parameters. Experimental trials performed during this study as well as a review of the literature demonstrated that tack levels as a function of process parameters can be generally understood considering the formation of strong Intimate Contact (IC), stress relaxation effects in the prepreg resin and the resin's cohesive strength. Perhaps layup temperature is the most sensitive process parameter that affects the prepreg tack and layup quality. By increasing the temperature, the resin viscosity decreases and a better IC is achieved, resulting in higher tack. Experimental observations, however, have reported a peak in the tack of prepregs and pressure sensitive adhesives as a function of temperature. This is due to the fact that reduction of resin viscosity (with increasing temperatures) is accompanied by the decline of resin strength. Consequently, at very high temperatures, a good contact is formed, whereas the lower strength of the resin results in cohesive failure within the prepreg (as opposed to interfacial failure at the prepreg-tool interface for lower temperatures). Layup speed in the AFP is inversely proportional to the contact time. As such, the lower speed means more time for IC formation, lower strain rates and more time for relaxation of stresses in the prepreg resin, all of which result in higher tack levels. Increasing compaction force, 
again, helps to establish a good IC and increases the prepreg tack. A peak is also observed for the tack as a function of compaction force, since the further increase of the force will result in redistribution of resin content and a contraction after the loading is released. Both these effects help the prepreg to detach from the tool surface (lower tack). Tack also has a peak around moderate values of relative humidity (50-60\%), which is generally attributed to the changes in resin viscosity and strength. Another important factor is the age of prepreg. As the material ages, crosslinking reaction occurs in the resin and the degree of cure increases. This will result in a stiffer material with lower tack. Using fresh prepregs within their shelf life helps in having a better quality layup. Other parameters such as tool's surface roughness, and, resin and tool surface energies also play a role in both establishing the IC and the amount of work of adhesion, however, their macroscopic effects on tack is not very well understood at the moment and further investigation is required.

The formation of wrinkles at the inside edge of the prepreg tow has been studied from both experimental [7-10] and modeling [11-13] perspectives. These approaches usually state a steering limit as the minimum radius that results in a defectfree layup. Experimental approaches are expensive, timeconsuming and increase material wastage since they are based on trial and error. On the other hand, modeling approaches take advantage of the incremental deposition of prepreg tows in the AFP, and assume that wrinkles are formed individually, in isolation from each other [12]. It has been noted that the assumption employed in the local modeling approach does not always follow the experimental observations [11]. Although these methods have gained some success, they both have limitations as stated. Recently, Hörmann [11] has recommended changing the modeling strategy for the future work by considering the global behavior of the prepreg tow, instead of using the local approach's assumption.

In the present paper, a framework for simulating the layup defects in the AFP is presented in the finite element software ABAQUS. The application of this approach for modeling the out-of-plane wrinkles at the inside edge of the steered prepreg tows is presented. The cohesive zone modeling technique with a bilinear traction-separation law is used to represent the prepreg tack at the prepreg-tool interface. In addition, it is found that the location of blister sites can be found using the simulations, which can be used to further explore the causes behind the blister formation. Preliminary results of the simulations are presented. Automated Fiber Placement trials are performed to validate the model. The trends and patterns of both wrinkles and blisters are found to be in very good agreement with experimental results.

\section{MODELING STRATEGY}

\section{A. Prepreg feeding and steering mechanisms}

The ABAQUS [14] model constitutes five parts: A rigid tool, a rigid bar which simulates the steering path, a compaction roller, a guide for the movement of prepreg tow, and finally the prepreg tow. The assembled model with the used boundary conditions are presented in Fig. 1. Appropriate boundary conditions and kinematic coupling constraints are

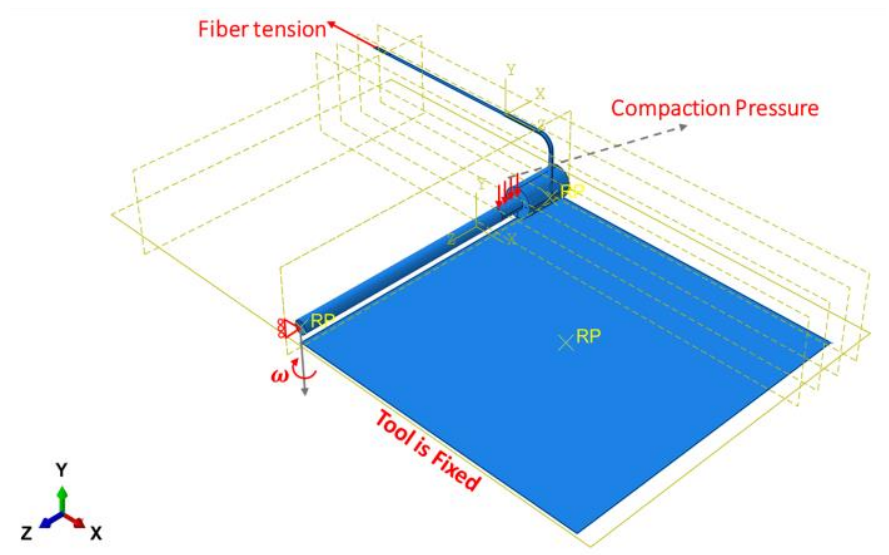

Figure 1. The assembled model.

created between the free end of the prepreg tow and the guide, to simulate the prepreg feeding mechanism. A light pressure simulating the compaction force is applied on top of the rigid bar, and a light force is applied to the free end of the tow to simulate the fiber tension which keeps the tows straight. A constant angular speed is applied to the free end of the rigid bar, such that it simulates the linear speed of the AFP head during the experiments.

\section{B. Prepreg tack}

Several different approaches are available for modeling fracture and delamination including continuum mechanics, fracture mechanics, XFEM and damage mechanics. For modeling the prepreg tack, an especial approach in the damage mechanics, called Cohesive Zone Modelling (CZM) is utilized [15]. Tack is merely intended to be modeled as an interfacial property; the delamination surface is known in the AFP process (at the prepreg-tool interface); and finally, since the location of out-of-plane wrinkles (and more generally, layup defects) are not known, the approaches requiring specification of an initial crack cannot be employed. Since CZM complies with all of the modeling requirements of prepreg tack, it has been chosen for the present study. CZM is well established in commercial finite element codes and more importantly, effective methods are available for characterizing the cohesive zone properties. They can be introduced into the ABAQUS model with the surfacebased cohesive zone modeling technique as a contact property. The cohesive contact is defined such that any node on the prepreg that comes into contact with the tool surface experiences the cohesive interactions. This modeling technique employs a bilinear traction-separation law to define the traction between the surfaces as a function of the separation between the surfaces. It is worthwhile to mention that in addition to the aforementioned desirable properties, viscoelastic material properties can be defined for the first part of the tractionseparation law which in conjunction with an exponential softening (damage evolution) can account for the ratedependent viscoelastic properties of prepreg tack.

Here, a bilinear traction-separation law for the CZM is employed which constitutes two linear sections (Fig. 2). The first section (elastic section) is defined through the slope of the line while the second part, corresponding to the damage evolution, requires a damage initiation criterion and a second 


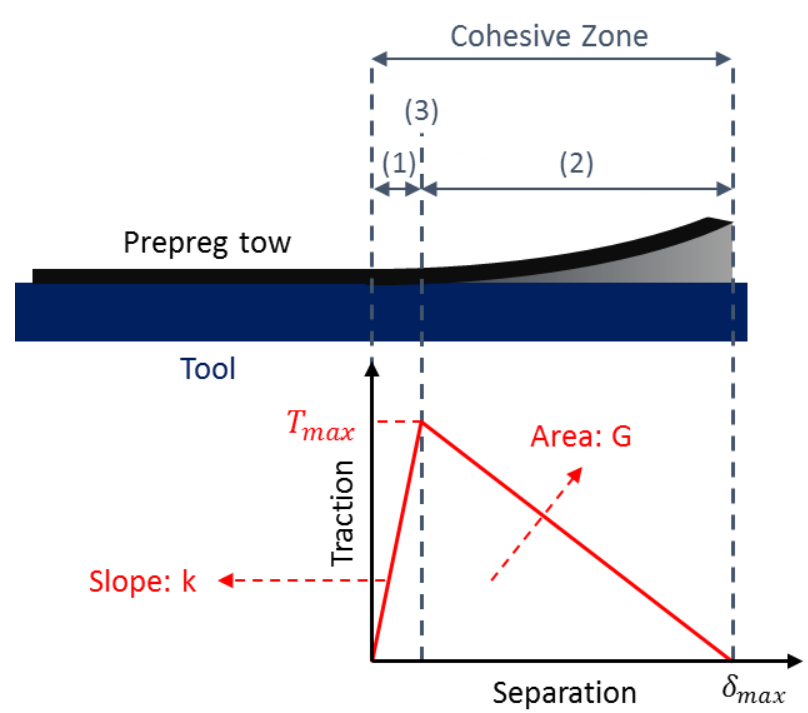

Figure 2. Bilinear traction separation law:

(1) Damage initiation (elastic), (2) Damage propagation (softening),

(3) Damage initiation criterion.

measure, for controlling the damage. Maximum nominal stress is chosen to establish the damage initiation and total fracture energy with linear softening is used to define the damage evolution.

From the fact that the maximum separation is, obviously, bigger than the separation at which the damage initiates, a simple relation should hold between the fracture energy (i.e. work of adhesion/tack), $G$, the maximum traction, $T_{\max }$, and stiffness (or the slope of the damage initiation part), $k$ :

$$
k>T_{\max }{ }^{2} / 2 G \text {. }
$$

\section{Solution method}

Several models with different mesh sizes were studied to find the appropriate trade-off between the computation time and resolution of the models. Abaqus / Explicit solver with full geometric nonlinearities was used to solve the model. The explicit solver can handle the nonlinearities that arise as a result of large deformations in the prepreg tow and material degradation in the traction-separation law, very well. It should be noted that on the contrary to the Standard solver, the value of stiffness, $k$, does matter in the Explicit solver. It is known that in order to avoid convergence problems, Standard solver treats the stiffness of the $\mathrm{CZ}$ in a manner similar to the penalty method, therefore the stiffness value should be large and it does not affect the final results significantly [14].

TABLE I.

PREPREG MECHANICAL PROPERTIES

\begin{tabular}{cccc}
\hline $\boldsymbol{E}_{\boldsymbol{1}}(\mathbf{G P a})$ & $\boldsymbol{E}_{\boldsymbol{2}}(\mathbf{M P a})$ & $\boldsymbol{G}_{12}(\mathbf{M P a})$ & $\boldsymbol{v}_{12}$ \\
\hline 31 & 0.046 & 3.025 & 0.2 \\
\hline
\end{tabular}

\section{MATERIAL CHARACTERIZATION}

The mechanical properties (except for the transverse modulus) of the uncured prepreg are experimentally measured in the previous work [13], using the tensile tests and bias extension tests. The transverse modulus was calculated using Puck's formula. These values are used in the present study since the same material was used in the AFP experimental trials. The material properties are summarized in table 1.

A commonly used method for measuring the unknown parameters required to fully characterize the prepreg tack using bilinear traction-separation laws is probe tack test method [4, 6]. This method was initially introduced in the Pressure Sensitive Adhesives industry to quantitatively measure the tackiness of adhesives and later was adopted to be used for measuring prepreg tack. In this testing method usually, a circular probe comes into contact with the prepreg at a controlled temperature and relative humidity, until a specified force is reached. Then it remains in contact with the prepreg for a given amount of time (dwell time), and subsequently, it is pulled away. During the retraction stage, force and displacement data are recorded to be further analyzed. Generally, the maximum recorded stress or the work of adhesion can be used to represent the quantitative value for tack.

Fig. 3 shows a typical normalized stress-displacement data obtained from the probe tack test (figure is reproduced from previous work [13]). As can be seen in this figure, the stiffness and maximum traction can be directly measured using probe test. The fracture energy (i.e. work of adhesion) can also be easily obtained by calculating the area under the traction data. These three parameters are sufficient for fully defining the bilinear CZ traction-separation law.

It should be noted that, although probe tack test provides a straightforward method for finding the required information for the model, its results might be hard to be related to the actual process parameters. The correlation between the AFP head's speed, on one hand, and the dwell time and probe speed (during both compression and retraction stages of probe test), on the other hand, is not clear. Moreover, the effects of temperature on the prepreg tack that is measured through probe test might be contrary to what actually occurs during the AFP. Increasing

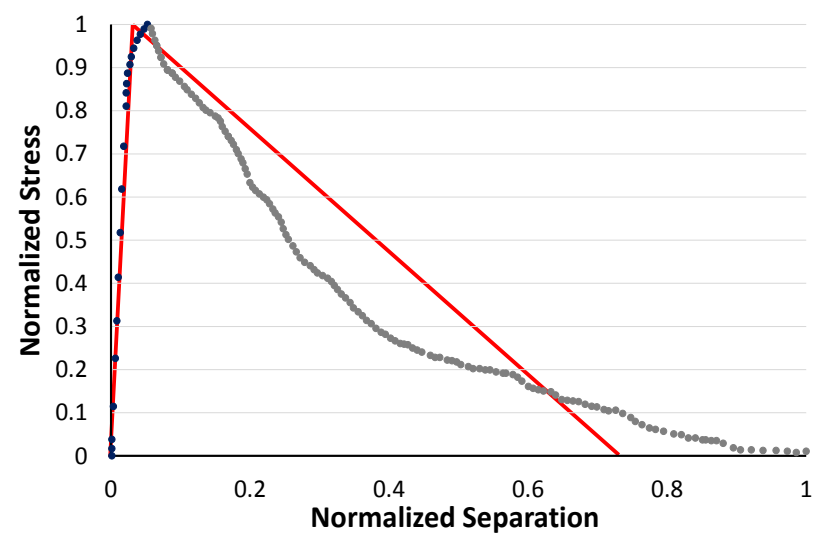

Figure 3. Typical data from probe tack test (reproduced from [13]), Data points: experimentally measured data, Red line: fitted bilinear traction-separation law. 


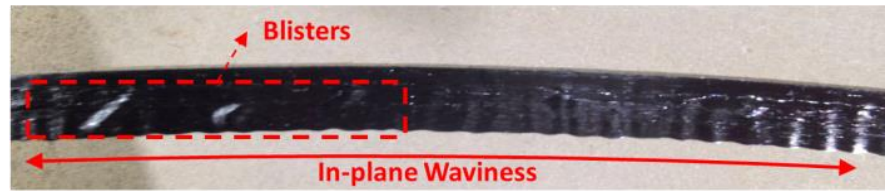

Figure 4. Blisters and in-plane waviness are appeared in the steered tows.

the temperature (which results in lowering the resin viscosity) in the probe test generally causes the resin to flows away from the probe surface. Consequently, lower tack results are usually recorded for increasing temperatures, while increasing temperature in the AFP clearly strengthens the interface (at least in the practical process range). Developing a more realistic approach for measuring prepreg tack with regards to AFP will remain for the future investigation. Here, available data in the literature $[6,13]$ that are in a close range of the experimental process parameters are used.

\section{RESULTS AND DISCUSSION}

AFP trials were performed using the $0.2 \mathrm{~mm}$-thick and 6.35 $\mathrm{mm}$-wide prepreg tows with the material properties presented in table 1. Tows are steered with the radius of $558.8 \mathrm{~mm}$ (22"). Results of the experiments and simulations are presented in this section.

In addition to the in-plane fiber waviness (Fig. 4) which is the most primitive result of length discrepancies between the inside and outside edges of a steered tow, a very commonly observed defects were blisters in the middle of the prepreg tows (Fig. 4 and 5). Initially, it was postulated that the blisters are occurring due to the high flow rate of the hot Nitrogen gas (heating system of the AFP). However, decreasing the flow rate proved to be ineffective in preventing their formation. During the simulations, it was found that in configurations similar to those in which blisters have appeared, several small zones are formed that have reached the damage initiation criterion but the damage has not progressed much. The results of the corresponding simulation are presented in Fig. 6 . The red color represents the areas where the damage initiation criterion of the traction-separation law is met. Those areas that have not completely buckled yet (i.e. the damage has not evolved $100 \%$ and consequently, wrinkles have not been formed) correspond to the blister zones and are marked with yellow circles. It can

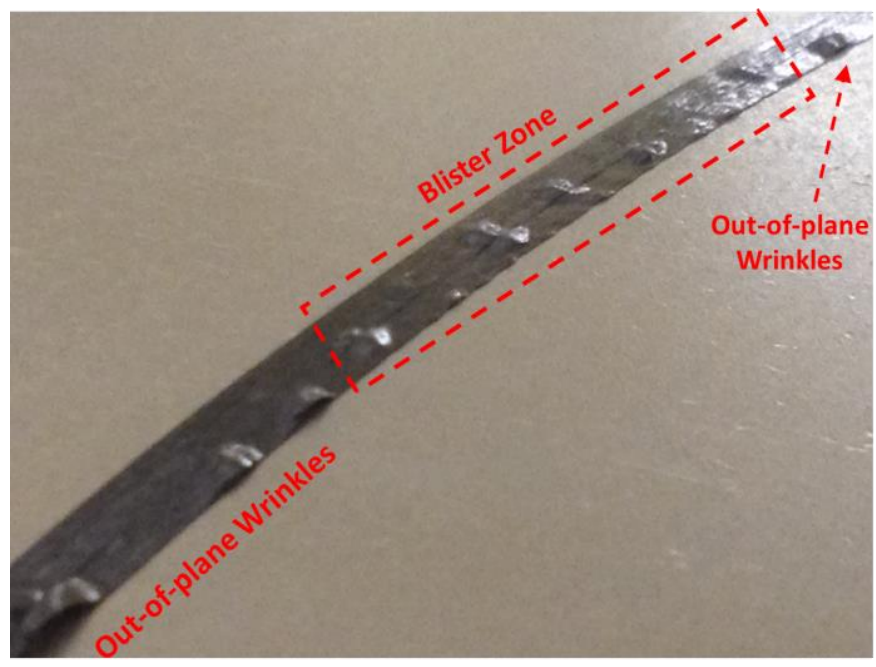

Figure 5. Blisters and out-of-plane wrinkles are appeared in the steered tows.

be observed from this figure that the wrinkles are clearly distinguished by the large deformation of the tow while the blister zones look intact (although they have a damaged interface which causes the blisters to appear in practice).

A qualitative comparison of Fig. 6 with the experimental results (Fig. 5) shows an excellent agreement, meaning that blisters are in fact mainly originating from the stress distribution resulted from fiber steering, and interface failure (i.e. not sufficient levels of tack) rather than other physical factors. Moreover, the pattern and frequency of appearance of out-of-plane wrinkles are also in good agreement between the simulation and experiments.

A commonplace result of the simulations is presented in Fig. 7. As it is demonstrated in this figure, the outside edge of the prepreg tow is under sever tensile stress. This tensile stress has two origins: the fiber tension force that is exerted on the free end of the tow which partly helps in keeping the loose tow straight (in reality this load is indeed induced in the prepreg tow in the delivery system), and the tensile stress that is induced as a result of fiber steering (bending stress). In the viewpoint of Fig. 7, two wrinkles are appeared. It is evident that compressive stress is built up at the inside edge of the tow

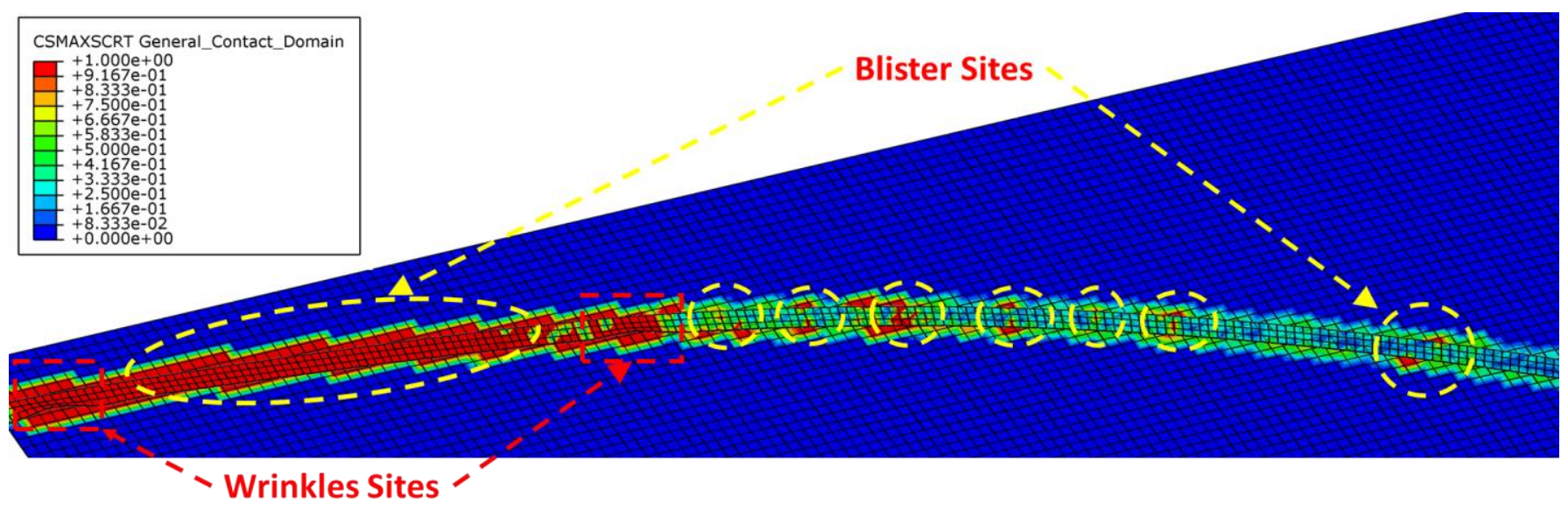

Figure 6. Simluation results: the damage initiation criteria. 


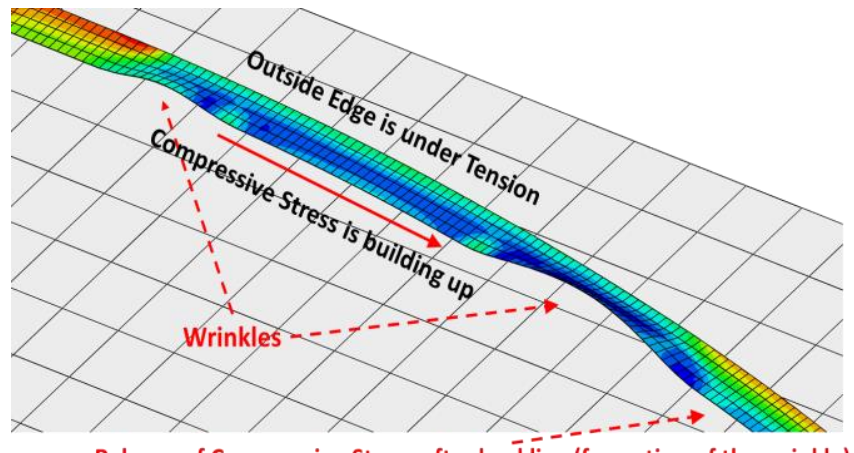

Release of Compressive Stress after buckling (formation of the wrinkle)

Figure 7. A commonplace result of the simulations.

between the two wrinkles. More importantly, small low-stress zones are visible in the vicinity of both wrinkles indicating that wrinkles are indeed acting as a stress relief mechanism.

\section{CONCLUSION}

A new global defect modelling approach for the layup process of Automated Fiber Placement is presented in the present work. A simple mechanism is created to simulate the feeding mechanism of the prepreg tow. The surface-based cohesive zone modelling technique using a bilinear tractionseparation law is used to model the prepreg tack. Application of the proposed approach for the challenging and commonplace case of fiber steering which is one of the major defective layup scenarios in AFP is discussed. Experimental AFP trials were performed and a very good agreement between the simulations and experimental results is found. It is shown that using the index representing the damage initiation criterion and the deformed shape of the prepreg tow, two of the major defects occurring during the tow steering namely, out-of-plane wrinkles and blisters, can be predicted and identified.

\section{ACKNOWLEDGMENT}

The authors would like to acknowledge the financial support from the Natural Sciences and Engineering Research Council of Canada (NSERC). Also, we would like to thank Mr. Jeffrey Fortin-Simpson of CONCOM's AFP laboratory for his help and assistance in sample preparations.

\section{REFERENCES}

[1] Dirk, H.-J.L., C. Ward, and K.D. Potter, The engineering aspects of automated prepreg layup: History, present and future. Composites Part B: Engineering, 2012. 43(3): p. 997-1009.

[2] Lozano, G.G., et al., A review on design for manufacture of variable stiffness composite laminates. Proceedings of the Institution of Mechanical Engineers, Part B: Journal of Engineering Manufacture, 2016. 230(6): p. 981-992.

[3] Ahn, K., et al., Analysis and characterization of prepreg tack. Polymer Composites, 1992. 13(3): p. 197-206.

[4] Dubois, O., J.-B. Le Cam, and A. Beakou, Experimental analysis of prepreg tack. Experimental Mechanics, 2010. 50(5): p. 599-606.

[5] Crossley, R., P. Schubel, and N. Warrior, The experimental determination of prepreg tack and dynamic stiffness. Composites Part A: Applied Science and Manufacturing, 2012. 43(3): p. 423-434.

[6] Wohl, C., et al., Tack Measurements of Prepreg Tape at Variable Temperature and Humidity. 2017.

[7] Nagendra, S., et al. Optimization of tow fiber paths for composite design. in Proceedings of the AIAA/ASME/ASCE/AHS/ASC 36th Structures, Structural Dynamics and Materials Conference, New Orleans, LA. 1995.

[8] Wiehn, M. and R. Hale. Low cost robotic fabrication methods for tow placement. in 47 the International SAMPE Symposium and Exhibition 2002. 2002.

[9] Chen, J., et al., Impact of layup rate on the quality of fiber steering/cutrestart in automated fiber placement processes. Science and Engineering of Composite Materials, 2015. 22(2): p. 165-173.

[10] Smith, R., et al., Limitations of processing carbon fibre reinforced plastic/polymer material using automated fibre placement technology. Journal of Reinforced Plastics and Composites, 2016. 35(21): p. 15271542 .

[11] Hörmann, P., Thermoset Automated Fibre Placement-on Steering Effects and Their Prediction. 2016: Verlag Dr. Hut.

[12] Matveev, M.Y., et al., Understanding the buckling behaviour of steered tows in Automated Dry Fibre Placement (ADFP). Composites Part A: Applied Science and Manufacturing, 2016. 90(Supplement C): p. 451456.

[13] Belhaj, M. and M. Hojjati, Wrinkle formation during steering in automated fiber placement: Modeling and experimental verification. Journal of Reinforced Plastics and Composites, 2018: p. 0731684417752872.

[14] ABAQUS, F., ABAQUS 6.14 Documentation. Dassault Systemes, Providence, RI, USA, 2014.

[15] Sauer, R.A., A survey of computational models for adhesion. The Journal of Adhesion, 2016. 92(2): p. 81-120. 